\title{
Bei Kälte Hautausschlag
}

Hautausschläge, Kopf- und Gelenkschmerzen, sobald die Temperaturen unter 15 Grad sinken: An diesen Symptomen leiden Patienten mit angeborener Kälte-Urtikaria. Eine Forschungsgruppe der Charité - Universitätsmedizin Berlin hat eine bisher unbekannte Form dieser entzündlichen Hauterkrankung entdeckt. Ihre Erkenntnisse erklären, warum herkömmliche Wirkstoffe bei manchen Betroffenen nicht helfen, und zeigen, was stattdessen wirksam sein könnte.

Bei vielen Patienten tritt die Kälte-Urtikaria spontan auf und ebbt nach einigen Jahren wieder ab. Bei einigen wenigen Betroffnen sorgt ein Gendefekt dafür, dass das Immunsystem auf kalte Temperaturen reagiert. Die Folge sind nicht nur Hautausschläge, sondern auch Zeichen einer körperweiten Entzündungsreaktion, beispielsweise Fieber oder Gelenkschmerzen. Unter Leitung von Dr. Karoline Krause, Campus Charité Mitte, haben Forschende eine neue erbliche Form der Kälte-Urtikaria entdeckt, die auf einer bisher unbekannten Mutation des sogenannten Faktor-XII-Gens beruht [1]. Für die neue Erbkrankheit wird daher die Bezeichnung „FACAS" (Faktor-XIIassoziiertes Kälte-induziertes autoinflammatorisches Syndrom) vorgeschlagen.

„Bei uns in der Klinik hat sich eine Familie vorgestellt, in der mindestens eine Person in jeder Generation von Geburt an über dieselben Symptome klagte“, erklärt Krause: „Wenn die Betroffenen eine halbe Stunde lang einer Temperatur von unter 15 Grad ausgesetzt waren, entwickelte sich ein brennender Hautausschlag, der besonders stark bei windigem und feuchtem Wetter ausgeprägt war und erst Stunden nach der Rückkehr in wärmere Räume zurückging."

Zusätzlich beschrieben die Patientinnen und Patienten Symptome wie Schüttelfrost, Kopf- und Gelenkschmerzen sowie Abgeschlagenheit. Im Gegensatz zu Menschen, die eine Kälte-Urtikaria spontan entwickeln, reagierten diese Patienten jedoch nicht auf den so genannten
Eiswürfel-Test. Ihre Symptome ließen sich außerdem nicht mit Antihistaminika behandeln, die bei spontaner Kälte-Urtikaria normalerweise helfen. Krause: „Wir suchten daher in der Erbinformation der betroffenen Familienmitglieder nach Mutationen, die wir bereits als Ursache für die erbliche Variante der Erkrankung kennen - allerdings vergeblich. Stattdessen fanden wir einen bisher nicht beschriebenen Defekt im Faktor-XIIGen."

\section{Defekt im Faktor-XII-Gen}

Die Forschungsgruppe konnte zeigen, dass dieser Defekt über eine Aktivierung des so genannten Kontaktsystems zur Freisetzung entzündlicher Botenstoffe führt - und so schließlich die Nesselsucht auslöst. „Interessanterweise kennen wir Fehler im FaktorXII-Gen eigentlich als Ursache für eine ganz andere Erkrankung, nämlich das hereditäre Angioödem", so Krause.

Beim hereditären Angioödem kommt es zu anfallsartigen und sehr schmerzhaften Hautschwellungen in tiefliegendem Gewebe. "Während die FACAS-Betroffenen also über Symptome einer angeborenen KälteUrtikaria klagen, sind die zugrunde liegenden Mechanismen ganz andere. Deshalb kommen für diese Patientinnen und Patienten Medikamente infrage, die wir normalerweise beim hereditären Angioödem verwenden." Tatsächlich verschwanden bei einer FACAS-Patientin die Kälte-induzierten Symptome weitgehend, sobald ihr der Wirkstoff Icatibant verabreicht wurde, der beim hereditären Angioödem im Akutfall eingesetzt wird.
Die aktuellen Studienergebnisse könnten dazu beitragen, die Krankheitsursache in bisher ungeklärten Fällen aufzudecken: Menschen mit angeborener Kälte-induzierter Urtikaria, bei denen bisher keine Ursache für die Erkrankung gefunden werden konnte und denen herkömmliche Medikamente wie Antihistaminika nicht helfen, können sich auf FACAS genetisch untersuchen lassen. „Zusätzlich planen wir eine kleine klinische Studie, um zu untersuchen, ob der Wirkstoff Lanadelumab den uns bekannten erwachsenen FACASBetroffenen nachhaltig Linderung verschafft", erläutert die Studienleiterin. Lanadelumab ist ein Antikörper, der nicht nur im Akutfall, sondern auch über einen längeren Zeitraum beim hereditären Angioödem verabreicht werden kann.

\section{Literatur}

1. Scheffel et al (2020) Cold-Induced Urticarial Autoinflammatory Syndrome Related to Factor XII Activation. Nat Commun 11:179. https://doi.org/10.1038/s41467-019-13984-8

Hinweis des Verlags. Der Verlag bleibt in Hinblick auf geografische Zuordnungen und Gebietsbezeichnungen in veröffentlichten Karten und Institutsadressen neutral.

hautnah $2020 \cdot 19: 60$

https://doi.org/10.1007/s12326-02000382-x

(c) Springer-Verlag GmbH Austria, ein Teil von Springer Nature 2020 